\author{
Sandra Ponce Ruiz',* \\ Teresa Sanclemente Boli² \\ Lidia Iñigo Cruces $^{3}$ \\ Consuelo Álvarez Lorenzo ${ }^{4}$ \\ Esther Les Morel| \\ Esperanza Zuriguel Pérez ${ }^{6}$
}

1. Enfermera Clínica. Unidad de Ortogeriatría. Hospital de Traumatología, Rehabilitación y Quemados Vall d'Hebron. Barcelona. España.

2. Supervisora. Unidad de Ortogeriatría. Hospital de Traumatología, Rehabilitación y Quemados Vall d'Hebron. Barcelona. España.

3. Enfermera. Unidad de Geriatría. Hospital Vall d'Hebron. Barcelona. España.

4. Adjunta de Enfermería. Hospital de Traumatología, Rehabilitación y Quemados Vall d'Hebron. Barcelona. España.

5. Supervisora. Hospital de Traumatología, Rehabilitación y Quemados Vall d'Hebron. Barcelona. España.

6. Doctora en enfermería. Coordinadora de investigación en enfermería. Hospitall Vall d'Hebron. Barcelona. España

\section{Perfil y satisfacción del patrón evacuador en pacientes hospitalizados en una unidad de ortogeriatría}

\section{RESUMEN}

Objetivos: Describir el perfil de los pacientes hospitalizados en la unidad de ortogeriatría de un hospital terciario en lo que respecta a su patrón evacuador e identificar la satisfacción de aquellos durante la hospitalización y los posibles motivos de insatisfacción. Metodología:

Estudio descriptivo y transversal sobre 200 personas mayores de 65 años ingresadas en la Unidad de Ortogeriatría del Hospital

Vall d'Hebron (Barcelona). Variables: sociodemográficas (edad y sexo), polifarmacia, consumo de fibra, consumo de laxantes previo y durante la hospitalización, ingesta hídrica, consumo de mórficos

durante la hospitalización, movilización precoz, estreñimiento crónico (Escala Roma III), satisfacción del patrón evacuador tanto en domicilio como durante la hospitalización, principales síntomas y factores desencadenantes de la posible insatisfacción. Resultados:

Predominio del sexo femenino (77\%) frente al masculino (23\%), edad media 83,92 ańos. Destaca polifarmacia (>5 fármacos) (63\%), consumo de opiáceos $(57,5 \%)$, automedicación de laxantes $(52,5 \%)$, insatisfacción del patrón evacuador durante el ingreso (65\%) respecto

al previo (38\%) y estreñimiento crónico (25\%) según criterios

Roma III. El síntoma más descrito por los pacientes es el esfuerzo excesivo para evacuar $(60 \%)$ y la demora en la respuesta de la llamada

(43,84\%). Se identificaron 8 factores que explican hasta el 61,25\% de la varianza total. Conclusión: El estudio ha permitido identificar los factores relacionados con el perfil de los pacientes hospitalizados en la Unidad de Ortogeriatría del Hospital Vall d'Hebron en lo que respecta a su patrón evacuador e identificar la satisfacción respecto al patrón de eliminación durante la hospitalización y los posibles motivos de insatisfacción.

PALABRAS CLAVE: Estreńimiento, anciano hospitalizado, fractura de fémur.

\section{ABSTRACT}

Objective: Describe the defecation pattern profile of patients hospitalized at Orthogeriatrics Unit of a tertiary Trauma Center, to evaluate defecation satisfaction during hospitalization and potential reasons for dissatisfaction. Methods: This is a cross-sectional descriptive study of 200 patients ( $>65$ years old) admitted to the Orthogeriatrics Unit of Hospital Vall d'Hebron. Evaluated variables incloude: sociodemographic date (age and sex), polypharmacy $(>5$ drugs), fiber intake, laxatives use before and during hospitalization, water intake, morphine intake during hospitalization, early mobilization, chronic constipation (Rome III scale), satisfaction of the evacuation pattern at home and during hospitalization, symptoms and trigger factors of their potential dissatisfaction. Results: Most of the patients were female (77\%) with a mean $(23 \%)$ age of 83.92 years. Among the enrolled patients, (63\%) take more than ( $>5$ drugs), $57.5 \%$ use opioid and $52.5 \%$ reported self-prescribed laxatives use $65 \%$ were dissatisfied with their evacuation pattern during admission compared to the previous one (38\%). According to Rome III criteria, $25 \%$ suffer from chronic constipation. The most reported symptom was the excessive straining $(60 \%)$ and the trigger factor for dissatisfaction was the delayed response to the call of sanitary teams (43.84\%). We obtained eight factors that explain up to $61.25 \%$ of the total variance. Conclusion: Due to the high rate of dissatisfaction reported by patients while, we believe it is necessary to carry out further research on new tools that improve constipation diagnosis, including specific criteria. Moreover, we recommend the implementation of an action algorithm could diminish the variability of therapeutic approaches in our clinical practice.

KEYWORDS: Constipation, hospitalized elders, hip fracture. 


\section{- INTRODUCCIÓN}

Los pacientes que ingresan por fractura de fémur en las unidades de ortogeriatría se caracterizan en general por su avanzada edad, polimedicación y pluripatología ${ }^{1}$, lo que les confiere esa característica de fragilidad ${ }^{2}$ o vulnerabilidad clínica que los acompañará durante su estancia hospitalaria y por las distintas transiciones asistenciales que deberán pasar, para garantizar la continuidad de los cuidados.

A estos factores se debe añadir la situación grave que comporta la fractura de fémur en sí, pues se trata de un proceso complejo que precisa de una hospitalización urgente, cirugía, sedación e inmovilidad, que puede suponer la descompensación de diversas patologías y la demora en la recuperación funcional ${ }^{1,3,4}$. Es por este motivo por el que la fractura de fémur representa un problema sociosanitario por su elevada morbimortalidad y los gastos que genera ${ }^{5}$. En nuestro país se calcula una incidencia anual aproximada de 200000 nuevas fracturas ${ }^{6}$, con una incidencia mayor en mujeres que en hombres, y se prevé que para el año 2040 el problema se triplique ${ }^{7}$.

La mortalidad hospitalaria de estos pacientes es de aproximadamente un $5 \%$ en función de su estancia media ${ }^{8,9}$, siendo el período postoperatorio uno de los más críticos de todo el proceso.

Entre las posibles complicaciones postoperatorias de la cirugía ortopédica de fractura de fémur en el anciano, el estreñimiento es una de la más prevalentes. Empeora los resultados de salud, la satisfacción de los pacientes e incrementa la carga asistencial y la estancia hospitalaria ${ }^{10}$.

Según la World Gastroenterology Organization Practice Guidelines ${ }^{11}$, el estreñimiento es un síntoma, no una enfermedad, y aunque los pacientes tienen distintas percepciones de los síntomas, lo suelen describir como una sensación de evacuación incompleta, un esfuerzo excesivo o intentos fallidos para defecar, deposiciones duras y/o disminución de la frecuencia de las deposiciones semanales ${ }^{10}$. De un modo más objetivo, según los criterios Roma III $^{10-12}$ se considera que un paciente sufre estreńimiento cuando en más del $25 \%$ de las deposiciones presenta dos o más de los siguientes síntomas: precisa de un esfuerzo excesivo, presenta heces duras, no logra una sensación confortable de desocupación rectal, tiene sensación de obstrucción en el área anorrectal, utiliza maniobras para facilitar la expulsión o evacua menos de tres veces por semana.

La diferencia entre criterios subjetivos y objetivos explica las discrepancias entre las tasas de prevalencia en el anciano. Hay estudios que señalan que alrededor del 20-30\% sufre estreńimiento a los 65 años, pero entre el 30\% y $50 \%$ de los que se autodefinen como estreñidos rondan los 75 años de edad ${ }^{13,14}$. Esta variabilidad respecto a la prevalencia también puede darse por la falta de parámetros concretos que determinen la normalidad del patrón de eliminación intestinal.

Centrándonos en el anciano hospitalizado con fractura de fémur, los factores de riesgo más relevantes son la inmovilidad que se produce ante la demora de la cirugía o en el retraso de la movilización postoperatoria, el consumo de ciertos fármacos o analgésicos opiáceos, hábitos higiénico-dietéticos inadecuados, la deshidratación secundaria a la hipovolemia, escasez de ingesta hídrica u omisión en la llamada de defecar, entre otros $^{10}$. Factores que pueden predisponer una alteración del patrón de eliminación intestinal respecto al previo y pueden desencadenar durante el ingreso graves complicaciones.

Dada la relevancia del problema en las personas mayores, para su manejo y correcto tratamiento es necesaria una valoración geriátrica ${ }^{2}$, anamnesis de su hábito previo deposicional ${ }^{10} \mathrm{y}$ un protocolo de actuación desde el momento del ingreso para minimizar sus posibles consecuencias.

La escasez de investigaciones y la ausencia de recomendaciones específicas sobre el estreñimiento de novo conlleva extrapolar las recomendaciones generales del estreñimiento crónico al proceso hospitalario. En este contexto nos hemos planteado el diseño de este estudio con la finalidad de describir la satisfacción del patrón evacuador de los pacientes ingresados en la Unidad de Ortogeriatría y dar a conocer la necesidad de unos criterios más específicos para su identificación y posterior solución.

\section{- OBJETIVOS}

Describir el perfil de los pacientes hospitalizados en la Unidad de Ortogeriatría del Hospital Vall d'Hebron, en lo que respecta a su patrón evacuador, e identificar la satisfacción del paciente durante la hospitalización y los posibles motivos de insatisfacción.

\section{- METODOLOGÍA}

Estudio descriptivo y transversal, llevado a cabo entre los meses de septiembre de 2017 y febrero de 2018. La población de estudio estuvo constituida por 200 pacientes ingresados en la Unidad de Ortogeriatría del Hospital Vall d'Hebron (Barcelona). Se consideraron criterios de inclusión pacientes mayores de 65 ańos, de ambos sexos y que se hallaran hospitalizados con una estancia mínima de una semana en la unidad tras sufrir una fractura de fémur. Se excluyó a los pacientes con una alteración cognitiva que dificultara la correcta anamnesis y la negativa a participar en el estudio y/o firmar el consentimiento informado.

Mediante un proceso de entrevista individual y revisión de la historia clínica se recogieron las siguientes variables: sociodemográficas (edad y sexo), polifarmacia ( $>5$ fármacos), consumo de fibra, consumo de laxantes previo y durante la hospitalización, ingesta hídrica, consumo de mórficos durante la hospitalización, movilización precoz, estreñimiento crónico (Escala Roma III), satisfacción del patrón evacuador tanto en domicilio como durante la hospitalización, principales síntomas y factores desencadenantes de la posible insatisfacción.

Para valorar de una forma más objetiva el estreñimiento crónico funcional se utilizaron los criterios Roma III descritos en el año 2006 ${ }^{10-12}$, que clasifican la patología funcional del aparato digestivo por síntomas. Según esta clasificación, se considera estreñimiento funcional cuando al menos dos de sus criterios se presentan al menos una vez por semana durante un periodo mínimo de 2 meses previos al diagnóstico.

La satisfacción del patrón evacuador se evaluó mediante una escala de tipo Likert, puntuando del 1 (muy insatisfecho) al 5 (muy satisfecho).

\section{- ANÁLISIS DE DATOS}

Se realizó un análisis descriptivo de todas las variables, donde las variables cualitativas fueron expresadas en frecuencias con porcentajes y las variables cuantitativas, con media y desviación estándar. Para establecer las relaciones entre las variables se aplicó análisis factorial por el método de extracción de componentes principales y el método de Kaiser-Meyer-Olkin (KMO). Para determinar la pertinencia se llevó a cabo el test de esfericidad de Barlett, y el análisis se estimó mediante comunalidades que mostraron el coeficiente de correlación lineal múltiple de cada variable con los factores. Por otra parte, se realizó una rotación de ejes por el método Varimax, método por el cual se realiza una rotación ortogonal que minimiza el número de variables que tienen saturaciones altas. El nivel de confianza fijado en las comparaciones fue del $95 \%$; se aceptaron como significativas las diferencias encontradas $p<0,05$. Se diseñó una base de datos específica para este estudio según el programa estadístico SPSS (Statistical Package for the Social Sciences) en su versión 22.0. 


\section{Consideraciones éticas}

El proyecto fue aprobado por el Comité de Ética de Investigación con Medicamentos del Hospital Vall d'Hebron. De acuerdo con la normativa vigente europea y nacional de protección de datos de carácter personal, los datos personales han sido los necesarios para cubrir los fines del estudio. Todos los participantes fueron informados de la voluntariedad y anonimato del estudio, solicitándose su consentimiento informado.

Tabla 1. Características de la muestra

\begin{tabular}{|c|c|c|}
\hline & $\mathbf{n}$ & $\%$ \\
\hline \multicolumn{3}{|c|}{ Consumo $>5$ fármacos (polifarmacia) } \\
\hline 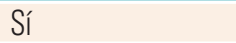 & 126 & $63 \%$ \\
\hline No & 74 & $37 \%$ \\
\hline \multicolumn{3}{|c|}{ Consumo de opiáceos durante la hospitalización } \\
\hline Sí & 115 & $57,5 \%$ \\
\hline No & 85 & $42,5 \%$ \\
\hline \multicolumn{3}{|c|}{ Movilización precoz (primeras 24 h) } \\
\hline Sí & 158 & $79,0 \%$ \\
\hline No & 42 & $21,0 \%$ \\
\hline \multicolumn{3}{|c|}{ Consumo de laxantes en el domicilio } \\
\hline Sí & 129 & $64,5 \%$ \\
\hline No & 71 & $35,5 \%$ \\
\hline \multicolumn{3}{|c|}{ Tipo de tratamiento laxante en domicilio } \\
\hline Prescripción médica & 22 & $11,0 \%$ \\
\hline Automedicación & 105 & $52,5 \%$ \\
\hline Terapias alternativas & 73 & $36,5 \%$ \\
\hline \multicolumn{3}{|c|}{ Tratamiento de laxantes durante el ingreso } \\
\hline Sí & 135 & $67,5 \%$ \\
\hline No & 65 & $32,5 \%$ \\
\hline \multicolumn{3}{|l|}{ Ingesta hídrica } \\
\hline $0-2$ vasos de agua & 10 & $5,0 \%$ \\
\hline $3-5$ vasos de agua & 168 & $84,0 \%$ \\
\hline$>6$ vasos de agua & 22 & $11,0 \%$ \\
\hline \multicolumn{3}{|l|}{ Consumo de fibra } \\
\hline Sí & 170 & $85,0 \%$ \\
\hline No & 30 & $15,0 \%$ \\
\hline \multicolumn{3}{|c|}{$\begin{array}{l}\text { Satisfacción sobre el patrón evacuador previo } \\
\text { Sí }\end{array}$} \\
\hline Sí & 124 & $62,0 \%$ \\
\hline No & 76 & $38,0 \%$ \\
\hline
\end{tabular}

Satisfacción sobre el patrón evacuador hospitalario (percepción de estreñimiento)

\begin{tabular}{|l|c|c|}
\hline Sí & 70 & $35 \%$ \\
\hline No & 130 & $65 \%$ \\
\hline Estreñimiento crónico (criterios Roma III) & & \\
\hline Sí & 50 & $25 \%$ \\
\hline No & 150 & $75 \%$ \\
\hline Descripción de los síntomas & & \\
\hline Sensación de evacuación incompleta & 42 & $21,0 \%$ \\
\hline Esfuerzo excesivo & 120 & $60,0 \%$ \\
\hline Dolor & 38 & $19,0 \%$ \\
\hline Factores desencadenantes (pacientes insatisfechos) & \\
\hline Falta de intimidad & 39 & $30 \%$ \\
\hline Demora en la respuesta de la llamada & 57 & $43,8 \%$ \\
\hline Incapacidad para defecar en la cama/pañal & 34 & $26,1 \%$ \\
\hline
\end{tabular}

\section{RESULTADOS}

\section{Descripción de los participantes}

La distribución de la población según las características sociodemográficas mostró que de los 200 pacientes incluidos en el estudio, predominaron pacientes del sexo femenino $(\mathrm{n}=154 ; 77 \%)$ frente al sexo masculino $(n=46 ; 23 \%)$. Respecto a la edad, el rango oscila entre 65 y 98 ańos, con un promedio de edad de $83,92( \pm 6,9)$ años.

Respecto a su patrón deposicional habitual antes de la hospitalización, un $46 \%$ de los pacientes refirieron de 2 a 3 evacuaciones a la semana, el $34 \%$ manifestaron de 4 a 5 evacuaciones a la semana, un 11\% realizaba deposiciones todos los días y un 9\% una vez por semana.

Durante la hospitalización se constató que un $33 \%$ realizaba de 2 a 3 evacuaciones por semana, el $49 \%$ de 4 a 5 evacuaciones a la semana, un $7 \%$ realiza deposiciones todos los días y el $11 \%$ una vez por semana.

En la tabla 1 se exponen las características de la muestra.

\section{Perfil y satisfacción del patrón evauador}

Los resultados de los índices de adecuación muestral KMO obtenidos $(0,568)$ y el nivel de significación de la prueba de esfericidad de Barlett $(0,000)$ apuntaron a la viabilidad del análisis factorial exploratorio con el fin de identificar las variables más relevantes.

El análisis factorial se realizó con base en la matriz de correlaciones (tabla 2), utilizando la extracción por componentes principales, y se interpretaron las cargas factoriales. Así se pudo determinar que variables tales como la satisfacción del patrón evacuador con un 0,905 explican en mayor proporción la varianza, mientras que variables como el sexo con un 0,394 apenas correlaciona con las restantes variables.

En la tabla 3 se puede observar que se han encontrado ocho factores de interés que explican hasta el $61,25 \%$ de la varianza total de todas las

Tabla 2. Comunalidades asociadas al estreñimiento

\begin{tabular}{|l|r|c|}
\hline \multicolumn{1}{|c|}{ Comunalidades } & Inicial & Extracción \\
\hline Satisfacción & 1,000 & 0,905 \\
\hline Movilización precoz & 1,000 & 0,843 \\
\hline Tratamiento con laxantes previo & 1,000 & 0,839 \\
\hline Estreñimiento (Roma III) & 1,000 & 0,822 \\
\hline Automedicación & 1,000 & 0,713 \\
\hline Edad (agrupada) & 1,000 & 0,678 \\
\hline Trastornos intestinales previos & 1,000 & 0,672 \\
\hline Opiáceos en planta & 1,000 & 0,638 \\
\hline Polifarmacia & 1,000 & 0,607 \\
\hline Tratamiento con laxantes en el hospital & 1,000 & 0,582 \\
\hline Ingesta hídrica & 1,000 & 0,541 \\
\hline Frecuencia de deposiciones al ingreso & 1,000 & 0,499 \\
\hline Esfuerzo excesivo & 1,000 & 0,478 \\
\hline Dolor al defecar & 1,000 & 0,477 \\
\hline Consumo de fibra & 1,000 & 0,472 \\
\hline Frecuencia deposiciones en el domicilio & 1,000 & 0,417 \\
\hline Sexo & 1,000 & 0,394 \\
\hline
\end{tabular}

Método de extracción: análisis de componentes principales. 
Tabla 3. Varianza total explicada

\begin{tabular}{|c|c|c|c|c|c|c|c|c|c|c|c|}
\hline \multicolumn{3}{|c|}{ Autovalores iniciales } & \multicolumn{3}{|c|}{$\begin{array}{c}\text { Sumas de extracción de cargas } \\
\text { al cuadrado }\end{array}$} & \multicolumn{3}{|c|}{$\begin{array}{c}\text { Sumas de rotación de cargas } \\
\text { al cuadrado }\end{array}$} & \multicolumn{3}{|c|}{$\begin{array}{c}\text { Sumas de rotación de cargas } \\
\text { al cuadrado }\end{array}$} \\
\hline Total & $\begin{array}{l}\text { Varianza } \\
(\%)\end{array}$ & $\begin{array}{l}\text { Porcentaje } \\
\text { acumulado }\end{array}$ & Total & $\begin{array}{l}\text { Varianza } \\
\qquad(\%)\end{array}$ & $\begin{array}{l}\text { Porcentaje } \\
\text { acumulado }\end{array}$ & Total & $\begin{array}{c}\text { Varianza } \\
(\%)\end{array}$ & $\begin{array}{l}\text { Porcentaje } \\
\text { acumulado }\end{array}$ & Total & $\begin{array}{l}\text { Varianza } \\
(\%)\end{array}$ & $\begin{array}{l}\text { Porcentaje } \\
\text { acumulado }\end{array}$ \\
\hline 2,598 & 12,989 & 12,989 & 2,091 & 10,455 & 10,455 & 2,091 & 10,455 & 10,455 & 2,091 & 10,455 & 10,455 \\
\hline 2,163 & 10,814 & 23,803 & 1,970 & 9,848 & 20,303 & 1,970 & 9,848 & 20,303 & 1,970 & 9,848 & 20,303 \\
\hline 1,596 & 7,978 & 31,780 & 1,665 & 8,327 & 28,631 & 1,665 & 8,327 & 28,631 & 1,665 & 8,327 & 28,631 \\
\hline 1,331 & 6,654 & 38,434 & 1,435 & 7,173 & 35,804 & 1,435 & 7,173 & 35,804 & 1,435 & 7,173 & 35,804 \\
\hline 1,251 & 6,254 & 44,688 & 1,420 & 7,099 & 42,903 & 1,420 & 7,099 & 42,903 & 1,420 & 7,099 & 42,903 \\
\hline 1,167 & 5,835 & 50,523 & 1,265 & 6,323 & 49,226 & 1,265 & 6,323 & 49,226 & 1,265 & 6,323 & 49,226 \\
\hline 1,112 & 5,559 & 56,082 & 1,218 & 6,089 & 55,315 & 1,218 & 6,089 & 55,315 & 1,218 & 6,089 & 55,315 \\
\hline 1,035 & 5,175 & 61,257 & 1,188 & 5,942 & 61,257 & 1,188 & 5,942 & 61,257 & 1,188 & 5,942 & 61,257 \\
\hline 0,956 & 4,778 & 66,035 & & & & & & & & & \\
\hline 0,932 & 4,659 & 70,694 & & & & & & & & & \\
\hline 0,929 & 4,646 & 75,340 & & & & & & & & & \\
\hline 0,818 & 4,088 & 79,428 & & & & & & & & & \\
\hline 0,740 & 3,699 & 83,127 & & & & & & & & & \\
\hline 0,700 & 3,501 & 86,628 & & & & & & & & & \\
\hline 0,670 & 3,350 & 89,978 & & & & & & & & & \\
\hline 0,633 & 3,165 & 93,142 & & & & & & & & & \\
\hline 0,564 & 2,818 & 95,960 & & & & & & & & & \\
\hline 0,495 & 2,477 & 98,437 & & & & & & & & & \\
\hline 0,199 & 0,995 & 99,433 & & & & & & & & & \\
\hline 0,113 & 0,567 & 100,000 & & & & & & & & & \\
\hline
\end{tabular}

Método de extracción: análisis de componentes principales.

variables seleccionadas. Los tres primeros y más importantes son los que se describen a continuación:

Factor I: las variables que componen mejor el primer factor son la satisfacción, la demora de la respuesta de llamada y el sexo.

Factor II: tratamiento laxante previo a la hospitalización, percepción de estreńimiento y sexo, aunque inversamente.

Factor III: movilización precoz, tratamiento laxante durante la hospitalización y polifarmacia.

\section{- DISCUSIÓN}

Los resultados obtenidos muestran a nivel sociodemográfico similitudes con otros autores, donde predomina el sexo femenino sobre el masculino ${ }^{15-17}$; y respecto a la edad, coinciden en que es más frecuente en el paciente anciano, sobre todo en los mayores de 75 años ${ }^{16,18,19}$.

Los criterios Roma III han sido nuestra herramienta de trabajo para diagnosticar el estreñimiento de forma más objetiva, considerando que los criterios de esta escala solo permiten identificar el estreńimiento crónico y no el estreñimiento de nueva aparición intrahospitalario. No obstante, según nuestro estudio, la prevalencia del estreńimiento subjetivo es superior al objetivo, dato que coincide con el estudio llevado a cabo por Garrigues et al. ${ }^{20}$. Quizá para el contexto hospitalario y para la corta estancia que habitualmente presentan estos pacientes, factores como la autopercepción del estreñimiento, la frecuencia del ritmo deposicional y el uso previo de laxantes se consideran los mejores parámetros definitorios.

El síntoma que mejor define el estreńimiento en la población de estudio es el esfuerzo defecatorio, y en nuestro estudio los resultados obtenidos son más robustos que en un estudio sueco poblacional ${ }^{11}$. Otros síntomas definitorios de estreñimiento en nuestros pacientes son la sensación de evacuación incompleta seguida de un menor porcentaje de dolor al defecar.

Aunque el estreñimiento tiene un origen multifactorial, en muchas ocasiones coexisten diferentes causas. En estas edades existen factores con mayor incidencia, como la polimedicación ${ }^{20}$, que frecuentemente padecen los ancianos. El consumo de más de 5 fármacos predispone al estreñimiento secundario por los efectos secundarios de los fármacos. En ese sentido, en nuestra Unidad de Ortogeriatría, con la incorporación del médico internista, se revalora la medicación habitual que toman los ancianos y se retira la que realmente no es necesaria o cuyos efectos secundarios sean mayores que los beneficios.

Para el correcto manejo del estreñimiento, existen unas medidas generales y otras farmacológicas. Las medidas no farmacológicas ${ }^{10,20}$ incluyen básicamente fomentar una correcta ingesta hídrica, aportar la fibra necesaria y, como hemos mencionado anteriormente, facilitar la movilidad. No obstante, en lo que se refiere a los hábitos higiénico-dietéticos más básicos, encontramos en la literatura científica que no es aconsejable 
aumentar la fibra alimentaria mientras el paciente no sea totalmente activo $^{21}$, por lo que esta recomendación no sería aconsejable durante la estancia hospitalaria de nuestros pacientes.

Por otra parte, aunque la gran mayoría de nuestros pacientes refieren consumir el aporte hídrico necesario, existen discrepancias en lo que respecta al aumento de la ingesta hídrica para evitar el estreńimiento a corto plazo. Se cree que el aumento de la ingesta hídrica produce un aumento de la diuresis y reblandecimiento de las heces, pero no de la frecuencia de las deposiciones ${ }^{22}$, por lo que esta recomendación carece de evidencia por el momento.

Dado que la inmovilidad prolongada favorece el estreńimiento, la recomendación esencial sería reanudar la movilidad cuanto antes ${ }^{10,21}$. En este sentido, en la Unidad de Ortogeriatría, una de las medidas fundamentales es la movilización precoz tras la cirugía, que se caracteriza por la sedestación y/o bipedestación del paciente dentro de las primeras 24 horas, dato que se refleja en nuestros resultados, donde un elevado porcentaje de nuestros pacientes toleran este tipo de intervención y les permite acceder al baño cuanto antes.

En determinadas situaciones, el tratamiento no farmacológico ${ }^{22,23}$ no será suficientemente efectivo y será necesario complementarlo con el farmacológico ${ }^{24,25}$. Nos llama la atención, el elevado porcentaje de pacientes que admiten automedicarse ${ }^{26,27}$, lo que lleva a plantearnos que quizá no le dan la importancia que realmente tiene y en consecuencia creen que pueden tratar este trastorno sin supervisión médica. Lo cierto es que un mal consumo de estos fármacos a largo plazo puede conducir a la cronificación del estreñimiento e interactuar de forma negativa con otros fármacos.

Existen otros factores, como son los psicológicos, que inciden negativamente en la satisfacción del paciente e inducen también al estreñimiento $^{23}$. Nuestro estudio sugiere que un alto porcentaje sufre una demora en la respuesta de la llamada, que quizá venga dada por la falta de intimidad que requiere algo tan personal como ir al baño, el mero hecho de compartir habitación o la incapacidad para evacuar en cama. Dado que la satisfacción del patrón evacuador disminuye mientras permane- cen ingresados, deberíamos incorporar medidas que, aunque supongan una sobrecarga en la actividad asistencial, mejorarían la satisfacción del paciente. Las posibles medidas son: respetar la rutina del hábito intestinal $^{21,23}$, favorecer ir al baño después de las comidas y proporcionar la intimidad necesaria.

Entre las limitaciones de esta investigación hay que considerar que el diseño trasversal ha facilitado la generación de hipótesis sobre los factores con el patrón de eliminación de los pacientes durante la hospitalización, si bien el uso de otras metodologías permitiría explorar más profundamente los posibles motivos de insatisfacción. Otra limitación que considerar está vinculada a la generalizabilidad de los resultados. Si bien la muestra estudiada es de un tamaño aceptable para un estudio de esta metodología, los resultados no podrán ser generalizados a otros ámbitos.

\section{- CONCLUSIÓN}

El estudio ha permitió identificar el perfil de los pacientes hospitalizados en la Unidad de Ortogeriatría del Hospital Vall d'Hebron, en lo que respecta a su patrón evacuador, e identificar la satisfacción acerca del patrón de eliminación durante la hospitalización y los posibles motivos de insatisfacción. Del perfil tipo destaca: ser mujer de edad avanzada, polifarmacia, consumo de opiáceos y laxantes durante la hospitalización, automedicación de laxantes en el domicilio y con una alteración de la satisfacción de su patrón evacuador durante la hospitalización.

Dada la elevada insatisfacción que perciben los pacientes mientras permanecen hospitalizados, creemos necesario realizar futuras investigaciones sobre nuevos criterios específicos y/o la elaboración de una herramienta para el diagnóstico del estreńimiento intrahospitalario. Además de la implantación de un algoritmo de actuación, la ausencia de este favorece la variabilidad para su abordaje terapéutico

\section{Conflicto de intereses}

Las autoras declaran no tener ningún conflicto de intereses.

\section{BIBLIOGRAFíA}

1. Guía catalana de Ortogeriatria-Geriatria HCSC [Internet]. [Acceso 5 de marzo de 2018]. Disponible en: https://geriatriahcsc.wordpress. com/2015/10/25/guia-catalana-de-ortogeriatria/

2. Fernández $E$, Estévez $M$. La valoración geriátrica integral en e anciano frágil hospitalizado: revisión sistemática. Gerokomos. 2013:24(1):8-13.

3. González Montalvo JL, Alarcón Alarcón T, Pallardo Rodil B, Gotor Pérez P. Mauleón Álvarez de Linera JL, Gil Garay E. Ortogeriatría en pacientes agudos (I). Aspectos asistenciales. Rev Esp Geriat Gerontol. 2008;43(4):239-51.

4. Kua J, Ramason R, Rajamoney G, Cong MS. Which frailty measure is a good predictor of early post-operative complications in elderly hip fracture patients? Arch Orthop Trauma Surg. 2016;136(5):63947.

5. Giannoulis D, Calori G, Gianoudis PV. Thirty-day mortality afte hip fractures: has anything changed? Eur J Orthop Traumatol 2016;26(4):365-70.

6. Etxebarria I, Careiro JL, Larrainzar R, Vaquero E, Roca L, Mesa M, et al. Guía SECOT-GEIOS en osteoporosis y fractura por fragilidad. Actualización. Rev Esp Cir Ortop Traumatol. 2015;59(6):373-93

7. Negrete J, Alvarado JC, Reyes RA. Fractura de cadera como factor de riesgo en la mortalidad en pacientes mayores de 65 años. Estudio de casos y controles. Acta ortop Mex. 2014;28(6):352-62.

8. Trazona FJ, Belenguer Á, Rovira E, Cuesta D. Orthogeratric care: improving patient outcomes. Clin Interv Aging 2016;11:843-56.

9. González JL, Alarcón T, Hormigo Al. Why do hip fracture patients die? Med Clin. 2011;137;355-60.

10. Verdejo C, Bixquert M, López JA, Mascaró J, Rey E, Vigara M. Guía de buena práctica clínica en geriatría. Estreñimiento en el anciano. Madrid: Sociedad Española de Geriatría y Gerontología; 2014

11. World Gastroenterology Organization Practice Guidelines. Estreñimiento: una perspectiva mundial. 2010 [Internet]. [Acceso $10 \mathrm{de}$ mayo de 2018]. Disponible en: http://www.worldgastroenterology. org/guidelines/global-guidelines/constipation/constipation-spanish

12. Gandell D, Straus SE, Bundookwala M, Tsui V Alibhai S. Treatment of constipation in older people. CMAJ. 2013;185(8):663-70.

13. Gallagher P, O'Mahony D. Constipation in old age. Best Pract Res Clin Gastroenterol. 2009;23:875-87.

14. Kellow JE, Drossman DA. Rome foundation diagnostic algorithm for common gastrointestinal symptoms: Bowel Disorders. Am Gastroenterol. 2010:105:775-85.

15. Serra J, Mascort J, Marzo M, Delgado S, Ferrándiz J, Díaz ER, et a Guia de práctica clínica sobre el manejo del estreñimiento crónico en el paciente adulto. Parte 1: Definición, etiología y manifestaciones clínicas. Gastroenterol Hepatol. 2017:40(3):132-41.

16. Schaafer DC, Cheskin LJ. Constipation in the elderly. Am Fam Physician. 1998:58:907-14

17. Hammond E. Some preliminary findings on physical complaints from a prospective study of 1.064 .004 men and women. Am J Pub Health. 1964;54:11-23

18. Mínguez M, Benages A, Mora F. Diagnóstico y Tratamiento del es treñimiento. En: Domínguez JE, ed. El paciente con síntomas digestives. 2. ${ }^{\text {a }}$ ed. Barcelona: Edika-Med. 2011. p.131-40.

19. Delgado Arós S, Soria MJ. Estreñimiento idiopático simple. En: Ponce J, ed. Tratamiento de las enfermedades gastroenterológicas. 3. ed. Barcelona: Elsevier-Doyma; 2011. p. 187-200.
20. Garrigues V, Galvez C, Ortiz V, Ponce M, Nos P, Ponce J. Prevalence of constipation: agreement among several criteria and evaluation of the diagnostic accuracy of qualifying symptoms and self-reported definition in a population-based survey in Spain. Am J Epidemiol. 2004:159:520-6.

21. Prevención de estreñimiento en persones mayores. Guía de buenas prácticas en Enfermería. Asociación Profesional de Enfermeras de Ontario. Proyecto de Guías de buenas prácticas en Enfermería [Internet]. [Acceso 24 de abril de 2018]. Disponible en: http://www. rnao.org/bestpractices

22. Mascaró J, Formiga F. Valoración y tratamiento del estreñimiento en el anciano. Rev Esp Geriatr Gerontol. 2006:41(4):232-9.

23. Guía práctica de actuación diagnostico-terapéutica en estreñimiento crónico. Fundación Española de aparato digestivo [Internet]. [acceso 19 de junio de 2018]. Disponible en: Guia estrenimiento_1230\%20(15).pdf

24. Mounsey A, Raleigh M, Wilson A. Management of Constipation in Older Adults. Am Fam Physician. 2015;92(6):500-4.

25. Juarranz M, Calvo MJ. Soriano Tratamiento del estreñimiento en el anciano. Medicina General. 2003;58:603-6 [Internet]. [Acceso el 10 de enero de 2018]. Disponible en: http://www.mgyfsemg.org/ medicinageneral/revista 58/pdf/603-606.pdf

26. Serra J, Mascort J, Marzo M, Delgado S, Ferrándiz J, Díaz ER, et al. Guía de práctica clínica sobre el manejo del estreñimiento crónico en el paciente adulto. Parte 2: Diagnóstico y tratamiento. Gastroenterol Hepatol. 2017:40(4):303-16.

27. Vacas E, Castellà I, Sánchez M, Pujol A, Pallarés MC, Balagué M. Automedicación y ancianos. La realidad de un botiquín casero. Aten Primaria. 2009:41(5):269-74. 